\title{
Angiosarcoma with Synchronous Cutaneous and Small Bowel Involvement: A Report of a Rare Presentation
}

\author{
Erin Reis ${ }^{1}$, Kadra Kalamaha ${ }^{1}$, Hongchen Jia ${ }^{2}$, Hermina D. Fernandes ${ }^{1}$ \\ 1. Internal Medicine, University of North Dakota School of Medicine and Health Sciences, Bismarck, USA 2. Pathology, \\ Sanford Health, Bismarck, USA
}

Corresponding author: Hermina D. Fernandes, hemeonc2016@gmail.com

\begin{abstract}
Angiosarcomas are mesenchymal neoplasms of vascular origin that represent approximately $2 \%$ of soft tissue sarcomas. We discuss the case of a 75-year-old female who had presented with a purple nodular rash along the bilateral nasolabial folds. Upon further work-up, she was diagnosed with angiosarcoma, with the confirmed involvement of multi-focal sites. These included biopsy proven sites of the face and duodenum along with the radiographic involvement of the lungs, liver, and osseous tissue. We report this unique presentation of a rare malignancy and the treatment course with radiation, paclitaxel, and bevacizumab. We also discuss the implications of her co-morbid liver cirrhosis and gastric antral vascular ectasia (GAVE) in terms of its influence on the development of the angiosarcoma and treatment response.
\end{abstract}

Received 09/04/2018

Review began 11/21/2018 Review ended 11/22/2018 Published 11/28/2018

๑) Copyright 2018 Reis et al. This is an open access article distributed under the terms of the Creative Commons Attribution License CC-BY 3.0., which permits unrestricted use, distribution, and reproduction in any medium, provided the original author and source are credited.
Categories: Internal Medicine, Radiation Oncology, Oncology

Keywords: angiosarcoma, cutaneous angiosarcoma, metastatic cancer, cirrhosis, gastric antral vascular ectasia, radiation, paclitaxel, bevacizumab

\section{Introduction}

Angiosarcomas are rare mesenchymal neoplasms of endothelial cells which fall under the umbrella of soft tissue sarcomas. The annual incidence of soft tissue sarcomas in the United States is estimated to be 10,000 cases per year [1]. Specifically, angiosarcomas are estimated to comprise about $1-2 \%$ of these soft tissue sarcoma cases [2]. Angiosarcomas can arise anywhere in the body, with about $60 \%$ of cases initially presenting in the skin as a cutaneous angiosarcoma [1]. The head and neck are the most common sites of cutaneous angiosarcoma, contributing 59.3\% in a large incidence analysis [2]. A number of risk factors for angiosarcoma have been elucidated in the literature including, but not limited to, radiation therapy, chronic lymphedema, environmental exposures (vinyl chloride, thorium dioxide, ultraviolet light, arsenic, anabolic steroids, foreign bodies), benign vascular lesions, arteriovenous fistulas, xeroderma pigmentosum, familial syndromes, retinoblastoma, and previous primary malignancy [3-5].

Here, we report a unique case of metastatic angiosarcoma with the involvement of the skin and small bowel, and have discussed the implications of comorbidities including cirrhosis and gastric antral vascular ectasia (GAVE).

\section{Case Presentation}

A 75-year-old Native American female presented to dermatology with a 'port wine' purple nodular rash on her nasolabial folds, of 12 months duration (Figure 1). There was no associated pruritus, burning, pain, or bleeding from the area. She did report drainage of some clear fluid when pressure was applied to the area. On physical exam, there were areas of raised, papular, nodular purple growth along the bilateral nasolabial folds. There was no evidence of drainage or infection. There were no oral lesions or skin lesions elsewhere on her body upon complete dermatological exam. Her medical history was remarkable for cirrhosis, deemed cryptogenic or secondary to non-alcoholic steatohepatitis (NASH) following evaluation by gastroenterology. Her medical history also included iron deficiency anemia secondary to GAVE, type II diabetes mellitus, hypertension, asthma, and endometrioid carcinoma of the ovary. 


\section{Cureus}

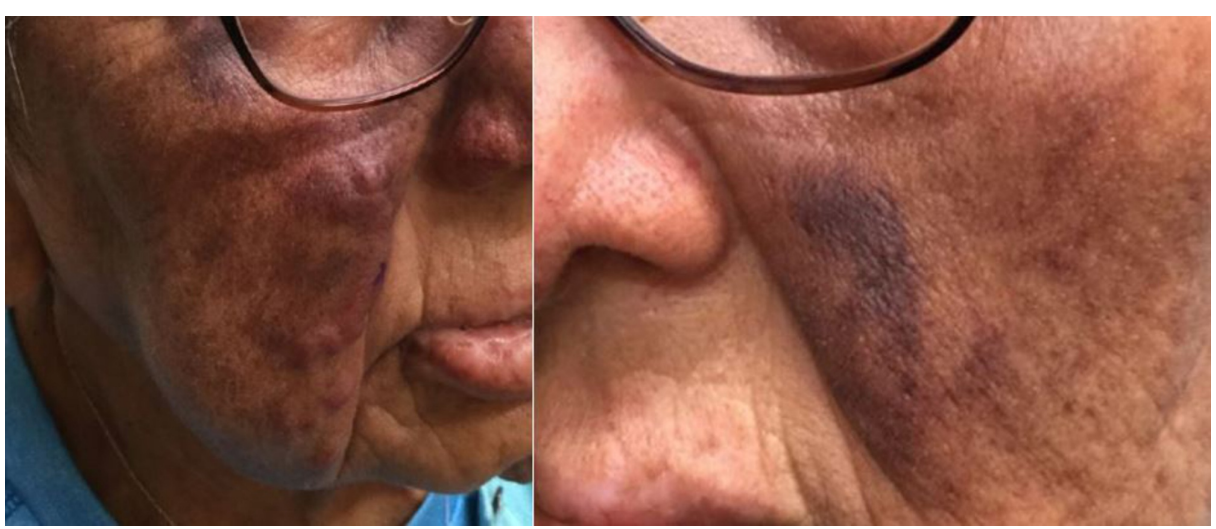

\section{FIGURE 1: Facial Lesion}

'Port wine' purple nodular rash on the patient's nasolabial folds

A 3-mm punch biopsy of the right nasolabial fold lesion demonstrated an atypical vascular lesion extending to the tissue margins. Sections revealed prominent vascular dilatation with papillary fragments and associated endothelial proliferation with cytologic atypia (Figure 2). A cluster of differentiation (CD)31 stain highlighted lesional cells, representing angiosarcoma.

A

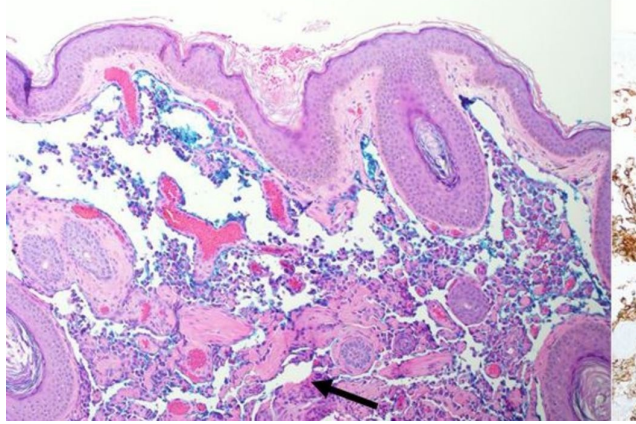

B

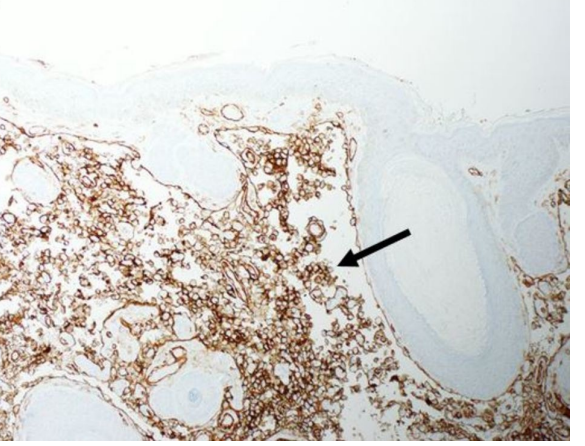

2.

\section{FIGURE 2: Skin Biopsy}

A. Vasoformative growth, with anastomosing channels in upper dermis (H\&E, x10). B. Tumor cells on the formalin-fixed section demonstrating strong membrane stain for CD31 (immunohistochemistry, x10).

She also underwent a surveillance gastrointestinal endoscopy due to her history of cirrhosis, and a duodenal ulcer was incidentally discovered. A biopsy was performed that revealed duodenal mucosa with ulceration and granulation tissue along with atypical, neoplastic proliferation of cells growing in sheets (Figure 3). Immunostains for erythroblast transformation-specific (ETS)-related gene (ERG) and friend leukemia integration 1 transcription factor (FLI1) were positive, confirming endothelial differentiation and thus consistent with angiosarcoma involving the duodenum.

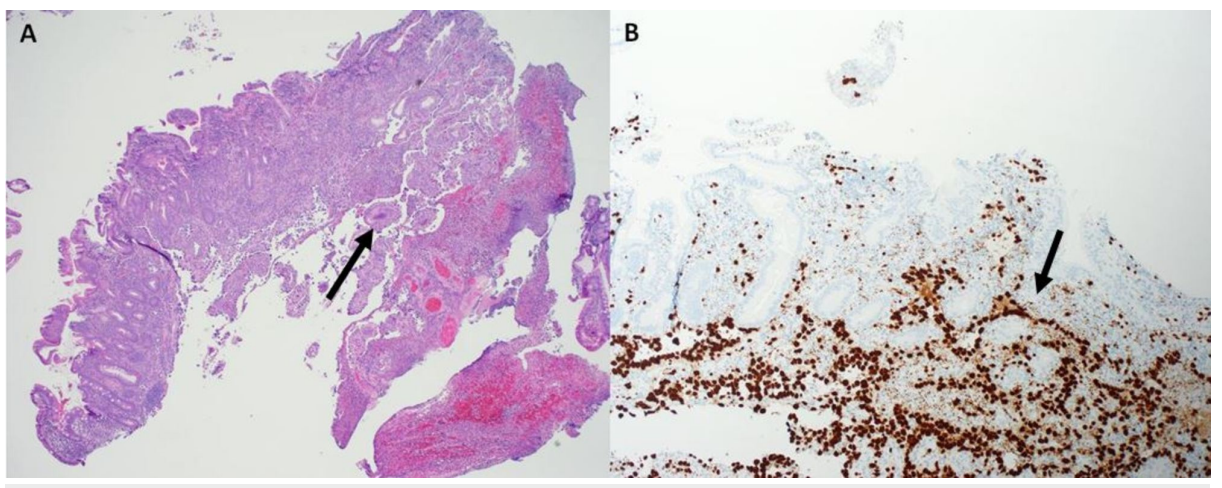

FIGURE 3: Duodenal Biopsy 


\section{Cureus}

A. Anastomosing vessels in lamina propria (H\&E, x4). B. Tumor cells of angiosarcoma demonstrating strong nuclear stain for ERG (immunohistochemistry, x4).

Staging evaluation was performed. Computed tomography (CT) imaging demonstrated right face superficial angiosarcoma without the invasion of deep tissues (Figure 4), bilateral lower lung nodules indeterminate for malignancy, and a $1.1 \mathrm{~cm}$ hypo-attenuated lesion of the right liver lobe indeterminate for malignancy. Positron emission tomography (PET) CT demonstrated hyper-metabolic activity in the face, consistent with the known lesion, and additionally hypermetabolic activity in the right scapular spine, distal sternum, pulmonary nodules of the left lung, right lobe of the liver, and T12 vertebral body (Figure 5). These findings suggested osseous and soft tissue metastases. A single liver lesion was biopsied and demonstrated changes consistent with chronic hepatitis and cirrhosis with no evidence of malignancy.

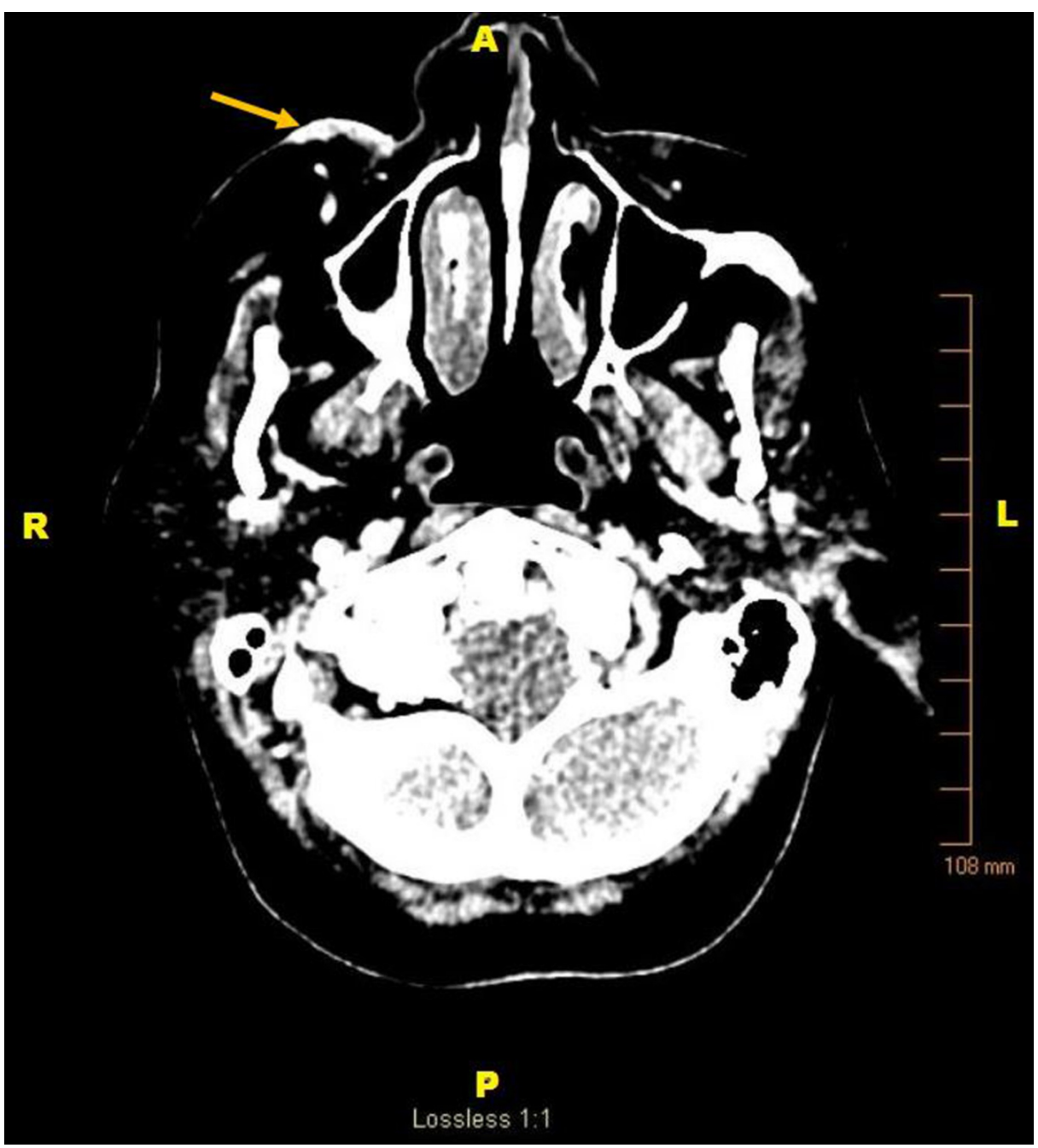

\section{FIGURE 4: Computed Tomography Head}

Computed tomography (CT) head with contrast pre-treatment showing (yellow arrow) cutaneous thickening and irregularity throughout the right face compatible with angiosarcoma. Legend: A - anterior; P - posterior; R -right; L - left 


\section{Cureus}

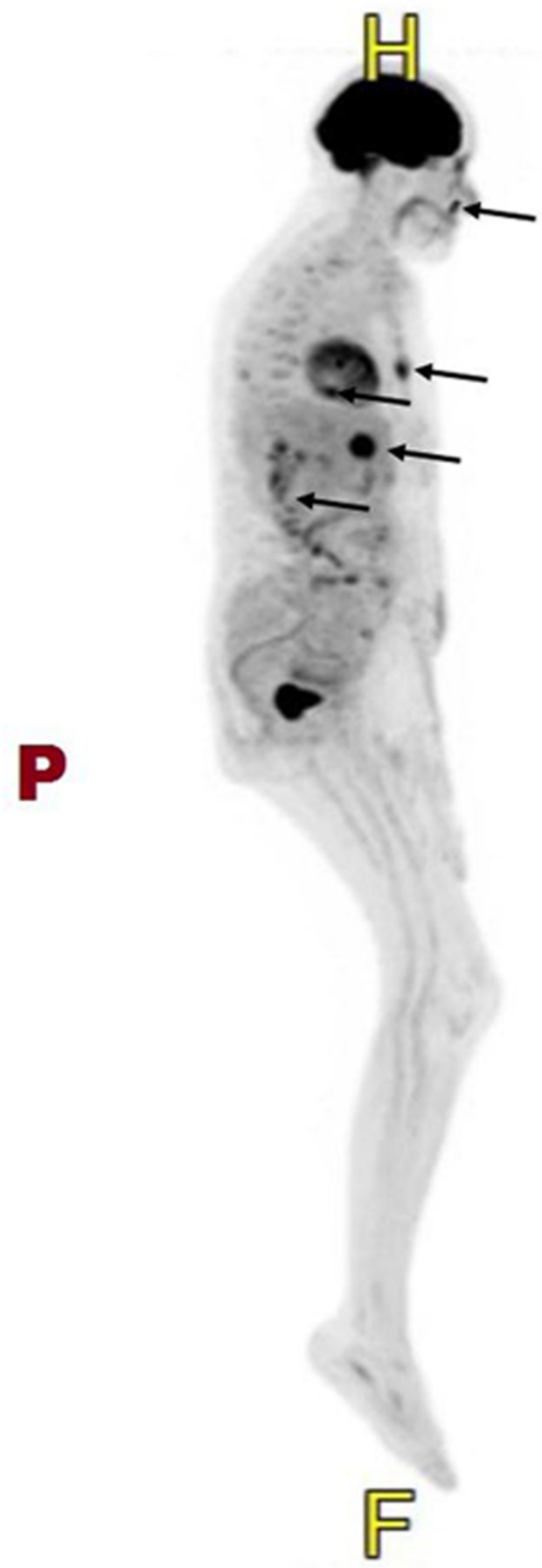

FIGURE 5: PET scan

PET scan pre-treatment showing increased uptake at sites of facial, sternal, pulmonary, liver and spinal lesions (arrows). Legend: A, anterior; P, posterior; H, head; F, foot

PET: Positron emission tomography

These findings confirmed the angiosarcoma of the face with multiple synchronous sites, including the duodenum. The patient was presented at the multidisciplinary tumor board where it was recommended that she receive palliative radiation for local control of the lesion on the face/nasolabial folds. She was also started on systemic therapy with intravenous paclitaxel as per the Phase II Trial of Weekly Paclitaxel for Unresectable Angiosarcoma (ANGIOTAX) study [6].

She experienced some treatment delay in between chemotherapy cycles due to radiation-induced 


\section{Cureus}

myelosuppression. Side effects were monitored closely, given her history of cirrhosis and baseline bicytopenia. She also experienced grade 2 cutaneous toxicity from radiation therapy including painful erythematous lesions in the mouth and over the lips as well as blepharitis. Post-radiation skin changes on the face eventually healed well, following completion of radiation. She did not experience any paresthesia with paclitaxel.

Imaging following two months of systemic chemotherapy revealed interval progression of hepatic and vertebral metastases (Figure 6).

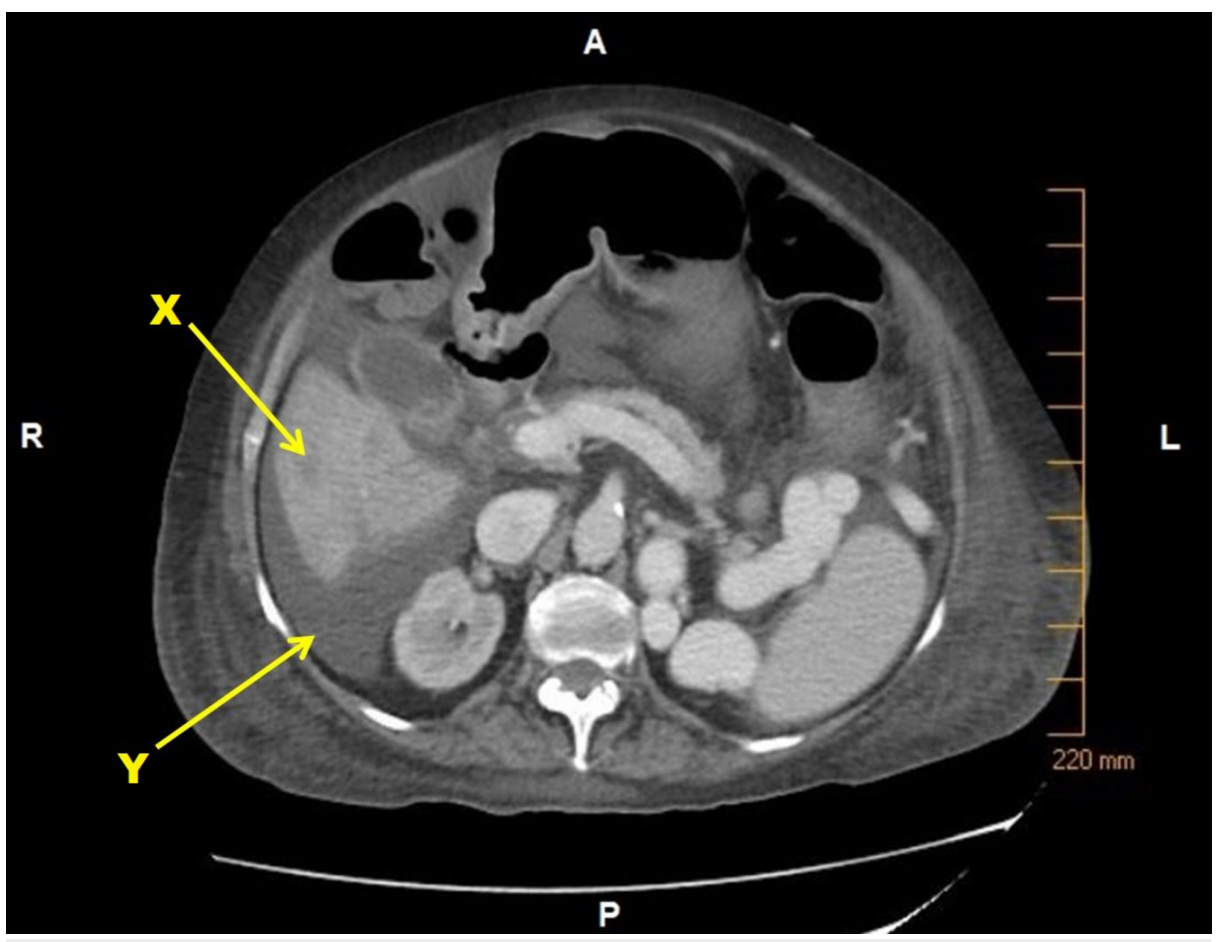

FIGURE 6: Computed Tomography (CT) Abdomen

Computed tomography (CT) abdomen with contrast post-treatment with first-line paclitaxel showing X. IIIdefined low-attenuating lesion in the inferior right hepatic lobe laterally measuring up to $2.0 \times 2.1 \mathrm{~cm}$ concerning for metastasis Y. Ascites. Legend: A - anterior; P - posterior; R - right; L - left

Due to disease progression, paclitaxel was discontinued and treatment was changed to second-line bevacizumab based on the available data from a phase II trial [7]. Following cycle 1 of bevacizumab, she experienced decompensated cirrhosis along with spontaneous bacterial peritonitis and ascites. Treatment was deferred due to multiple hospitalizations in the next few weeks due to decompensated cirrhosis. The patient and her family then elected to transition to hospice due to worsening quality of life. She died six months following her diagnosis of angiosarcoma with complications from decompensated liver cirrhosis.

\section{Discussion}

Angiosarcomas are tumors arising in either the blood vessels or lymphatics. The scalp and face are the most common sites of origin [8]. They are aggressive in nature, have a high metastatic potential, and are associated with poor prognosis. A large meta-analysis found the five-year survival rate for angiosarcomas of the scalp and face to be $33.5 \%$ [9]. It has been estimated that $16-46 \%$ of angiosarcoma cases present with metastatic disease or as multi-focal lesions, with hematogenous spread to lung and brain to be most common [1,9]. Factors associated with better prognosis for cutaneous angiosarcomas include having an age lower than 70 years, a lower number of affected regions, lack of metastatic disease, involvement of the face versus the scalp, negative margins upon resection, tumor size less than $5 \mathrm{~cm}$, histologic growth demonstrating greater than $80 \%$ solid areas, and programmed death-ligand 1 (PD-L1) expression [8-11].

The involvement of the small bowel is even more uncommon and is associated with worse outcomes as compared to cutaneous angiosarcomas. A recent literature review of previously published data examining small bowel angiosarcomas concluded that survival with intestinal involvement is less than one year and confers an extremely poor prognosis [12]. A retrospective analysis of 66 patients with small intestine angiosarcomas demonstrated a mean survival time of 30 weeks from diagnosis, with death usually associated with uncontrollable gastrointestinal bleeding or respiratory failure [13]. 
In our patient, due to synchronous presentation, it is not possible to determine whether the cutaneous lesion or the duodenal lesion was the primary tumor. A retrospective review of the published literature suggests that a majority of small bowel angiosarcomas are primary tumors. It was also noted that the likelihood of metastases to the intestine was the highest when the primary sites of the tumor were the skin or aorta [13]. The presence of epithelioid variant cells in the cutaneous biopsy from the face, as in our case, is more likely to be associated with angiosarcoma of the soft tissues as opposed to an intestinal primary angiosarcoma [3]. The tendency of these tumors to be multifocal, with an ability to develop at any site and a variety of presentations, make it challenging to ascertain the primary site of the tumor, as in this case.

Some data have suggested that the benign vascular lesions may be a risk factor for malignant transformation into angiosarcoma [4]. In this case, the patient's history of GAVE could be implicated in the development of angiosarcoma, although these could also be related to portal hypertension from her cirrhosis.

Our patient also had cirrhosis deemed secondary to NASH or cryptogenic cirrhosis upon prior investigation. Exposure to vinyl chloride has been implicated as a risk factor for hepatic angiosarcoma and cirrhosis [14-15]. It is reasonable to consider the possibility of angiosarcoma arising in the liver and coexisting in the context of cirrhosis. This incidence of hepatic angiosarcoma coexisting in the background of cirrhosis is especially high when both are linked to vinyl chloride exposure [14]. It is unclear whether the patient had a prior history of vinyl chloride exposure. She was also found to have hepatic tumor deposits radiographically during the course of her disease, which raises the possibility of a hepatic source of the tumor.

In the context of treatment, complete excision with wide margins is the treatment of choice for locally invasive disease. However, cutaneous angiosarcomas have been associated with high rates of local recurrence [11]. Due to the propensity for local infiltration, radiation therapy is also utilized to achieve local disease control [7]. Metastatic angiosarcomas have been proven to be sensitive to paclitaxel, with the median progression-free survival of 5 to 7.6 months [16]. Weekly and every three-week schedules have also been described with varying success [16]. Molecular targeted therapies such as sorafenib have been reported to delay the progression of the disease with a progression-free survival of $31 \%$ at six months [16]. Imatinib has also been reported to show a modest benefit with a progression-free survival benefit of $20 \%$ at three months [16]. Another phase II study demonstrated that anti-angiogenic agents like bevacizumab had activity against tumor with a median time to progression of 26 weeks [7].

\section{Conclusions}

Though the incidence is low, their aggressive behavior and poor prognosis make angiosarcomas a threatening diagnosis that should be identified and treated as soon as possible. The risk factors that should alert a clinician to look for an angiosarcoma are many. They include radiation therapy, chronic lymphedema, environmental exposures, ultraviolet light, arsenic, anabolic steroids, foreign bodies, benign vascular lesions, and arteriovenous fistulas. This malignancy is also commonly associated with xeroderma pigmentosum, familial syndromes, retinoblastoma, and previous primary malignancy. The prognosis for a metastatic disease remains in evolution with the availability of multiple agents for targeted therapy such as imatinib, sorafenib, and bevacizumab.

\section{Additional Information}

\section{Disclosures}

Human subjects: Consent was obtained by all participants in this study. Conflicts of interest: In compliance with the ICMJE uniform disclosure form, all authors declare the following: Payment/services info: All authors have declared that no financial support was received from any organization for the submitted work. Financial relationships: All authors have declared that they have no financial relationships at present or within the previous three years with any organizations that might have an interest in the submitted work. Other relationships: All authors have declared that there are no other relationships or activities that could appear to have influenced the submitted work.

\section{Acknowledgements}

The authors would like to thank the patient and her family.

\section{References}

1. Patt JC, Haines N: Soft tissue sarcomas in skin: presentations and management . Semin Oncol. 2016, 43:413418. 10.1053/j.seminoncol.2016.02.026

2. Rouhani P, Fletcher CDM, Devesa SS, Toro JR: Cutaneous soft tissue sarcoma incidence patterns in the U.S.: an analysis of 12,114 cases. Cancer. 2008, 113:616-627. 10.1002/cncr.23571

3. Ali JA, Ko HH, Owen D, Steinbrecher UP: Epithelioid angiosarcoma of the small bowel. Gastrointest Endosc. 2006, 64:1018-1021. 10.1016/j.gie.2006.04.020

4. Young RJ, Brown NJ, Reed MW, Hughes D, Woll PJ: Angiosarcoma. Lancet Oncol. 2010, 11:983-991. 10.1016/S1470-2045(10)70023-1 
5. Albores-Saavedra J, Schwartz AM, Henson DE, et al.: Cutaneous angiosarcoma: analysis of 434 cases from the surveillance, epidemiology, and end results program, 1973-2007. Ann Diagn Pathol. 2011, 15:93-97. 10.1016/j.anndiagpath.2010.07.012

6. Penel N, Bui BN, Bay JO, et al.: Phase II trial of weekly paclitaxel for unresectable angiosarcoma: the ANGIOTAX study. J Clin Oncol. 2008, 26:5269-5274. 10.1200/JCO.2008.17.3146

7. Agulnik M, Yarber JL, Okuno SH, et al.: An open-label, multicenter, phase II study of bevacizumab for the treatment of angiosarcoma and epithelioid hemangioendotheliomas. Ann Oncol. 2013, 24:257-263. 10.1093/annonc/mds237

8. Dettenborn T, Wermker K, Schulze H, Klein M, Schwipper V, Hallermann C: Prognostic features in angiosarcoma of the head and neck: a retrospective monocenter study. J Craniomaxillofac Surg. 2014, 42:1623-1628. 10.1016/j.jcms.2014.05.002

9. Shin JY, Roh SG, Lee NH, Yang KM: Predisposing factors for poor prognosis of angiosarcoma of the scalp and face: systematic review and meta-analysis. Head Neck. 2017, 39:380-386. 10.1002/hed.24554

10. Shimizu A, Kaira K, Okubo Y, et al.: Positive PD-L1 expression predicts worse outcome in cutaneous angiosarcoma. J Glob Oncol. 2016, 3:360-369. 10.1200/JGO.2016.005843

11. Ishida Y, Otsuka A, Kabashima K: Cutaneous angiosarcoma: update on biology and latest treatment . Curr Opin Oncol. 2018, 30:107-112.

12. Ni Q, Shang D, Peng H, et al.: Primary angiosarcoma of the small intestine with metastasis to the liver: a case report and review of the literature. World J Surg Oncol. 2013, 11:242. 10.1186/1477-7819-11-242

13. Li R, Ouyang ZY, Xiao JB, et al.: Clinical characteristics and prognostic factors of small intestine angiosarcoma: a retrospective clinical analysis of 66 cases. Cell Physiol Biochem. 2017, 44:817-827. $10.1159 / 000485345$

14. Frullanti E, La Vecchia C, Boffetta P, Zocchetti C: Vinyl chloride exposure and cirrhosis: a systematic review and meta-analysis. Dig Liver Dis. 2012, 44:775-779. 10.1016/j.dld.2012.02.007

15. Zheng YW, Zhang XW, Zhang JL, et al.: Primary hepatic angiosarcoma and potential treatment options . J Gastroenterol Hepatol. 2014, 29:906-911. 10.1111/jgh.12506

16. Penel N, Marréaud S, Robin YM, Hohenberger P: Angiosarcoma: state of the art and perspectives . Crit Rev Oncol Hematol. 2011, 80:257-263. https://doi.org/10.1016/j.critrevonc.2010.10.007 